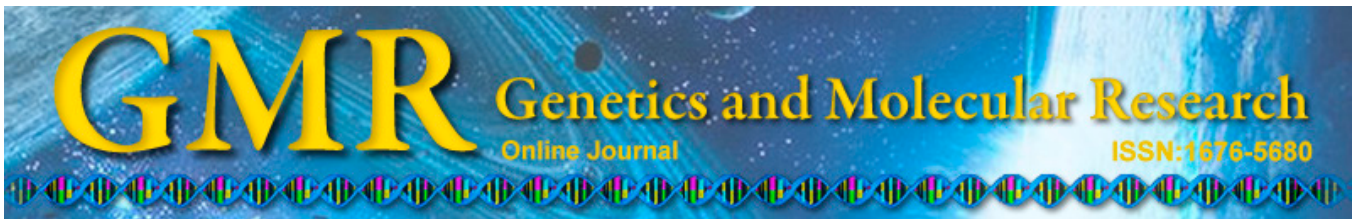

\title{
Comparison of high-intensity focused ultrasound therapy under nasal endoscopy guidance versus first-line drug treatment in patients with persistent allergic rhinitis
}

\author{
G.F. Feng, Z.L. Han, F. Wang, B.C. Sun, Z.Y. Dai, S.Z. Yang and C.Y. Zhou \\ Department of Otolaryngology Head and Neck Surgery, \\ First Affiliated Hospital of Chinese PLA General Hospital, Beijing, China \\ Corresponding author: C.Y. Zhou \\ E-mail: chengyongzhoucn@163.com \\ Genet. Mol. Res. 14 (3): 9865-9871 (2015) \\ Received December 10, 2014 \\ Accepted May 18, 2015 \\ Published August 19, 2015 \\ DOI http://dx.doi.org/10.4238/2015.August.19.20
}

\begin{abstract}
The aim of this study was to determine whether highintensity focused ultrasound (HIFU) therapy under nasal endoscopy guidance could provide better efficacy and safety in patients with persistent allergic rhinitis (PAR) than the first-line drugs recommended by the World Health Organization. A total of 120 adult patients with PAR were randomly divided into 2 groups $(\mathrm{N}=60$ each). One group underwent HIFU therapy under nasal endoscopy guidance using an ultrasound rhinitis therapeutic machine. The other group served as the control group and was treated with corticosteroid nasal spray and oral cetirizine hydrochloride. All patients underwent follow-up treatment for 1 year, after which the efficacy and safety were evaluated. There was no significant difference between the two groups $(P>0.05)$ in the total effective rate. Moreover, no complications such as nasal adhesion, septal perforation, mucosal atrophy, and hyposmia were observed, indicating that HIFU was as effective as the first-line drug treatments
\end{abstract}


recommended by the World Health Organization for symptom relief in PAR patients. The treatment efficacy, repeatability, safety, economical aspects, ease of performance, and few complications of HIFU therapy strongly suggest that HIFU should be routinely incorporated into clinical practice.

Key words: Persistent allergic rhinitis; Nasal endoscopy; High-intensity focused ultrasound; $\mathrm{H}_{1}$-antihistamines; Intranasal corticosteroids; Ultrasound's thermal effect

\section{INTRODUCTION}

Allergic rhinitis (AR) is a global health problem. For the last half century, the prevalence of AR has continuously increased in most parts of the world, and the identification of an effective and safe treatment for AR is still an important topic for current clinical AR investigations. AR is a chronic inflammatory disease of the nasal mucosa, which is triggered by the release of immunoglobulin (Ig) E-mediated mediators (mainly histamine) when the sensitive individual is exposed to allergens. This high incidence of refractory otorhinolaryngological disease results from the activities of a variety of immune cells and cytokines. The main symptoms include sneezing and nasal congestion, dripping, and itching. Over nearly half a century, AR has increased continuously in most parts of the world (Adsule and Misra, 2010).

In 2001, the diagnostic guidelines "Allergic Rhinitis and its Impact on Asthma" (ARIA) were published by the World Health Organization (WHO) (Bousquet et al., 2001). These guidelines indicated that AR is a global health problem, which usually coexists with asthma, and also stressed that AR is a risk factor for asthma. According to the ARIA guidelines, AR is generally classified as intermittent or persistent, and more specifically classified as mild, moderate, or severe, which is based on the symptom severity and impact on the quality of life (Gunhan et al., 2011). The main principles of AR management include avoiding allergens, drug therapy, immune therapy, and education. According to the ARIA classification system, the WHO has recommended a step-by-step treatment approach for AR (Bousquet et al., 2008). However, drug therapies can induce side effects, while immune therapy generally has poor compliance and is expensive. Therefore, an important topic of current clinical AR investigations is the identification of an effective and economical treatment for $\mathrm{AR}$, associated with minimal or no side effects.

\section{MATERIAL AND METHODS}

\section{Clinical information}

A total of 120 adult patients ( 72 males and 48 females) diagnosed with persistent allergic rhinitis (PAR) according to the AR diagnostic criteria established by the 2004 Otorhinolaryngology Society of the Chinese Medical Association (2005, Lanzhou, China) were analyzed. The mean patient age was 36.8 years (range $=16-67$ years), and the course of disease ranged from 1 to 30 years. This study was conducted in accordance with the declaration of Helsinki and with approval from the Ethics Committee of First Affiliated Hospital of Chinese PLA 
General Hospital. Written informed consent was obtained from all participants.

Symptoms at onset included intermittent nasal itching, congestion, continuous sneezing, and watery dripping. Nasal examination showed pale nasal mucosa, edema, hyperemia, and swelling. In the exacerbation period, nasal secretion smears showed the presence of eosinophils $(+)$ and allergen skin prick tests showed responses of at least one or above $(++)$. No recruited patients had nasal polyps or severe nasal septum deviation.

The patients were randomly divided into 2 groups of 60 patients each. One group received high-intensity focused ultrasound (HIFU) therapy under nasal endoscopy guidance using an ultrasound rhinitis therapeutic machine (Seapopinna, Chongqing, China). The other group served as the control and received corticosteroid nasal spray and oral cetirizine hydrochloride. The therapeutic effects were observed and recorded.

\section{Methods}

Patients in the HIFU group were sprayed with $1 \%$ ephedrine on both sides of the nose while lying in the supine position in order to shrink the nasal mucous membrane. Subsequently, under nasal endoscopy guidance, cotton patches containing $2 \%$ tetracaine were inserted into the bilateral nasal cavities for 5 min twice, in order to anesthetize the surface of the nasal septum, inferior turbinate, middle turbinate, and agger nasi. Under endoscopy, an ultrasound probe was secured closely to the nasal mucus and a single-scan line was scanned twice using the following ultrasound rhinitis therapeutic machine parameters: frequency, $10 \mathrm{MHz}$; sound power, III; scan rate, $2-6 \mathrm{~mm} / \mathrm{s}$; and scan line interval, $4 \mathrm{~mm}$. The scan was focused on the bilateral agger nasi, the anterior half of the inferior turbinate (the distribution area of the anterior ethmoidal nerve and posterior inferior nasal nerve), and the distribution area of the anterior ethmoidal nerve in the nasal septum. The scanning of any particular area lasted no more than $2 \mathrm{~s}$, and the total scan time was within 6-10 min, depending on the condition of the nasal cavity and the severity of the disease.

After the procedure, in order to reduce mucosal edema and prevent adhesion, the patients were treated alternately with two kinds of nasal drops (1\% furosemide ephedrine and peppermint oil). The patients were instructed to clean the nasal secretions for 3 days after the procedure and were examined once a week for 3 weeks in order to prevent adhesion between the inferior turbinate and nasal septum. If the symptoms were not reduced after three visits, the treatment was repeated. Similarly, if the disease relapsed, the treatment was also repeated. After 1 year of follow-up treatment, no complications such as nasal adhesion, septal perforation, mucosal atrophy, and hyposmia were observed.

The control patients received corticosteroid nasal spray (one spray on each side, twice daily; AstraZeneca, Switzerland) and oral cetirizine hydrochloride $(10 \mathrm{mg} / \mathrm{night}$; Yangtze Pharmaceutical Company, China). Upon symptom relief, the oral antihistamines were discontinued and the frequency of nasal corticosteroid spray was reduced.

\section{Evaluation of treatment efficacy}

The treatment efficacy was evaluated according to "The Principles of Diagnosis and Treatment of Allergic Rhinitis and Recommended Plan" established by the Otorhinolaryngology Society of the Chinese Medical Association (2005, Lanzhou, China) after 1 year of follow-up treatment. The established classification criteria for the symptom and physical sign 
scores are defined in Tables 1 and 2, respectively.

The effective rate of PAR was calculated based on the total symptom and sign scores before and after the treatment using the following formula: effective rate $=$ (total score before treatment - total score after treatment) / total score before treatment x 100\%. Effective rates $\geq 66 \%, 65-26 \%$, and $\leq 25 \%$ were considered remarkably effective, effective, and ineffective, respectively.

Table 1. Classification criteria for symptom scores.

\begin{tabular}{lccll}
\hline Score & Sneezing $^{\mathrm{a}}$ & Nose dripping $^{\mathrm{b}}$ & Nose congestion & Nasal itching \\
\hline 0 & None & None & None & None \\
1 & $3-5$ & $\leq 4$ & Congestion when breathing & Intermittent itching \\
2 & $6-10$ & $5-9$ & Intermittent mouth breathing & Tolerable itching \\
3 & $\geq 11$ & $\geq 10$ & Interactive mouth breathing almost all day & Intolerable itching \\
\hline
\end{tabular}

${ }^{a}$ Daily number of sneezes, bdaily incidence of nasal dripping.

Table 2. Classification criteria for physical sign scores.

\begin{tabular}{ll}
\hline Score & Status of the inferior turbinate, nasal septum, and middle turbinate \\
\hline 1 & $\begin{array}{l}\text { The inferior turbinate is mildly swollen, but the nasal septum and middle turbinate are still visible } \\
\text { The inferior turbinate and nasal septum (or the bottom of the nasal cavity) have either closed against each other } \\
\text { or only a small gap remains } \\
\text { The inferior turbinate, nasal septum and the bottom of nasal cavity are closed tightly. The middle turbinate is not } \\
\text { visible, or mucous polypoid and polyp formation can be seen }\end{array}$ \\
\hline
\end{tabular}

\section{Statistical analysis}

The $\chi^{2}$ test was used to evaluate the treatment differences.

\section{RESULTS}

The treatment results are shown in Table 3. It was demonstrated that there was no significant difference between the two groups $(\mathrm{P}>0.05)$ in the total effective rate, suggesting that the two treatment methods had similar efficacy.

Table 3. Comparison of efficacy in patients with mild or medium-severe symptoms.

\begin{tabular}{|c|c|c|c|c|c|c|c|c|}
\hline \multirow[t]{2}{*}{ Groups } & \multirow[t]{2}{*}{$\mathrm{N}$} & \multicolumn{3}{|c|}{ Mild symptoms } & \multicolumn{3}{|c|}{ Medium-severe symptoms } & \multirow[t]{2}{*}{ Total effective rate } \\
\hline & & $\begin{array}{c}\text { Remarkably } \\
\text { effective }\end{array}$ & Effective & Non-effective & $\begin{array}{l}\text { Remarkably } \\
\text { effective }\end{array}$ & Effective & Non-effective & \\
\hline Control & 60 & 9 & 4 & 0 & 36 & 3 & 8 & $86.7 \%$ \\
\hline HIFU & 60 & 10 & 2 & 0 & 39 & 3 & 6 & $90.0 \% *$ \\
\hline
\end{tabular}

*Non-significant difference by the $\chi^{2}$ test; $\mathrm{P}>0.05$.

\section{DISCUSSION}

PAR is an IgE-mediated, type I allergic reaction. Exposing individuals who have an idiosyncratic immune system to antigens can trigger the production of the corresponding $\operatorname{IgE}$ antibodies, which can lead to a sensitized status. When these individuals are repeatedly ex- 
posed to similar antigens, these antigens, together with the participation of complements, will combine with IgE molecules and bind to IgE receptors on mast cell membranes. In turn, this interaction stimulates the mast cells to produce a series of biochemical changes on the cell membrane, leading to the degranulation and release of a series of inflammatory mediators such as histamine, slow-reactive substances, and bradykinins (Layton et al., 2011; Braido et al., 2012; von Bernus et al., 2012). Consequently, these mediators bind to their respective receptors on the blood vessels, glands, and nerve endings on the nasal mucus and later induce significant inflammatory reactions. These reactions include contraction of resistance vessels (pale nasal mucus), blood volume expansion (light blue nasal mucus and congestion), increased capillary permeability (mucosal edema), excessive glandular secretion (increased nasal dripping), and enhanced nerve sensitivity (continuous sneezing). Further studies have indicated that allergic reactions consist of two phases: acute-phase and late-phase reactions. The main difference between these two phases is that acute-phase reactions result from the effects of $\operatorname{IgE}$, while late-phase reactions are mediated mainly by the major histocompatibility complex (Cingi and Ozlugedik, 2010; Navarro et al., 2011).

The first-line PAR treatments include intranasal corticosteroids and $\mathrm{H}_{1}$-antihistamines, both of which are recommended by the WHO (Cox and Wallace, 2011). Second-line treatments include anti-leukotrienes, cromones, decongestants, and anti-cholinergics; in the present study, only the first-line treatments were used.

Glucocorticosteroids produce strong anti-inflammatory effects by reducing the release of cytokines and chemotactic factors. These drugs could inhibit inflammatory reactions in multiple stages. As a result, they are considered to be the most effective drugs for treating allergic rhinitis and other allergic diseases (Barnes, 1999; Pasquali et al., 2006; Bousquet et al., 2012). Although the safety of intranasal corticosteroids has been well recognized, the possibility of undesirable local and systemic side effects should not be ignored. Local side effects include dry nose, nose bleeding, and nasal septal perforation. Systemic side effects include inhibition of the hypothalamus-pituitary-adrenal axis, stunting developmental growth in children, and ocular complications (Wu et al., 2001; Dávila et al., 2006). These systemic side effects remain a serious concern, which might limit the wide application of intranasal corticosteroids in clinical practice, and are the subjects of ongoing intensive research.

On the other hand, $\mathrm{H}_{1}$-antihistamines $\left(\mathrm{H}_{1}\right.$ receptor antagonists) work by blocking the effects of histamine, a major mediator of AR, and therefore play an important role in AR treatment (Ribeiro de Andrade et al., 2010; Aypak et al., 2013). The pharmacological mechanism of antihistamine activity involves competitive binding to $\mathrm{H}_{1}$ receptors and blocking of the biological effects of histamine. This blockade can eliminate many symptoms caused by mast cell granules, and this is one of the reasons why $\mathrm{H}_{1}$-antihistamines have been used as the firstline drug for the treatment of AR (Vovolis et al., 2013). The biggest drawbacks of antihistamines are their central sedation and anti-cholinergic effects, which have partly limited its wide application in clinical practice. Although second-generation antihistamines cause barely any drowsiness, there have been some reports regarding cardiac side effects, such as QT prolongation, which may lead to serious cardiac arrhythmia (Higaki et al., 2012). Fortunately, this vital cardiac side effect has rarely been observed in clinical practice; however, when prescribing AR therapies, clinicians should pay special attention to other comorbid diseases of AR patients, such as organic heart disease, cardiac arrhythmia, and electrolyte imbalance (Adsule and Misra, 2010; Ciebiada et al., 2011; Cox et al., 2012).

On the other hand, focused ultrasound technology can focus an ultrasound beam on 
diseased tissues inside the body (i.e., therapeutic target tissue) for a short period. Through the mechanical, thermal, and cavitation effects (mainly thermal effects) of ultrasound, the local temperatures can be increased to $70^{\circ}-100^{\circ} \mathrm{C}$, resulting in protein coagulation and spotty necrosis (Dávila et al., 2006). Herein, using a Seapopinna ultrasound rhinitis therapeutic machine, high-energy ultrasound was focused on the lower layer of the nasal mucus, which contains a large number of immune cells, plasma cells, serous mucous glands, nerves, and blood vessels, thereby causing protein coagulation and spotty necrosis.

The HIFU efficacy is the results of several different mechanisms. As inflammatory cells and cells with active metabolism are very sensitive to ultrasound, HIFU can directly damage local invasive immune cells and decrease the cell numbers, which in turn can reduce the levels of cytokines and inflammatory mediators released from these cells. HIFU can also induce the degranulation of mast cells, similar to immunotherapy. Moreover, HIFU can destroy the parasympathetic ganglion cells and substance P nerve fibers in the deep layer of the nasal mucus. As a result of this process, the excitability of the cholinergic nerves is reduced, and, at the same time, vasoactive peptides are released, thus alleviating vasodilatation and glandular secretion. All above effects are equivalent to the therapeutic effect of anti-cholinergic drugs. In addition, HIFU can directly block blood vessels or lead to thrombus formation through damaging of the vascular endothelial cells that partially or completely block the vessels. The results of these actions are equivalent to the effects of decongestants, which include reducing plasma exudation and relieving nasal edema.

Through the present clinical study, it was demonstrated that HIFU was as effective as the first-line drug treatment (recommended by the WHO) for symptom relief of PAR patients. Therefore, the treatment efficacy, repeatability, safety, economical aspects, ease of performance, and few complications of HIFU therapy strongly suggest that HIFU should be routinely incorporated into clinical practice.

\section{Conflicts of interest}

The authors declare no conflict of interest.

\section{REFERENCES}

Adsule SM and Misra D (2010). Long term treatment with montelukast and levocetirizine combination in persistent allergic rhinitis: review of recent evidence. J. Indian Med. Assoc. 108: 381-382.

Aypak C, Türedi Ö, Solmaz N, Yıkılkan H, et al. (2013). A rare adverse effect of montelukast treatment: ecchymosis. Respir. Care 58: e104-e106.

Barnes PJ (1999). Therapeutic strategies for allergic diseases. Nature 402: B31-B38.

Bousquet J, Van Cauwenberge P, Khaltaev N, Aria Workshop Group, et al. (2001). Allergic Rhinitis and its impact on asthma. J. Allergy Clin. Immunol. 108: S147-S334.

Bousquet J, Khaltaev N, Cruz AA, Denburg J, et al. (2008). Allergic Rhinitis and its Impact on Asthma (ARIA) 2008 update (in collaboration with the World Health Organization, GA(2)LEN and AllerGen). Allergy 86: 8-160.

Bousquet J, Schünemann HJ, Samolinski B, Demoly P, et al. (2012). Allergic Rhinitis and its Impact on Asthma (ARIA): achievements in 10 years and future needs. J. Allergy Clin. Immunol. 130: 1049-1062.

Braido F, Riccio AM, Rogkakou A, Massacane P, et al. (2012). Montelukast effects on inflammation in allergic rhinitis: a double blind placebo controlled pilot study. Eur. Ann. Allergy Clin. Immunol. 44: 48-53.

Ciebiada M, Gorska-Ciebiada M, Barylski M, Kmiecik T, et al. (2011). Use of montelukast alone or in combination with desloratadine or levocetirizine in patients with persistent allergic rhinitis. Am. J. Rhinol. Allergy 25: e1-e6.

Cingi C and Ozlugedik S (2010). Effects of montelukast on quality of life in patients with persistent allergic rhinitis. Otolaryngol. Head Neck Surg. 142: 654-658. 
Cox L and Wallace D (2011). Specific allergy immunotherapy for allergic rhinitis: subcutaneous and sublingual. Immunol. Allergy Clin. North Am. 31: 561-599.

Cox L, Calderón M and Pfaar O (2012). Subcutaneous allergen immunotherapy for allergic disease: examining efficacy, safety and cost-effectiveness of current and novel formulations. Immunotherapy 4: 601-616.

Dávila I, Sastre J, Bartra J, del Cuvillo A, et al. (2006). Effect of H1 antihistamines upon the cardiovascular system. J. Investig. Allergol. Clin. Immunol. 1: 13-23.

Gunhan K, Unlu H, Yuceturk AV and Songu M (2011). Intranasal steroids or radiofrequency turbinoplasty in persistent allergic rhinitis: effects on quality of life and objective parameters. Eur. Arch. Otorhinolaryngol. 268: 845-850.

Higaki T, Okano M, Makihara S, Fujiwara T, et al. (2012). Early interventional treatment with intranasal corticosteroids compared with postonset treatment in pollinosis. Ann. Allergy Asthma Immunol. 109: 458-464.

Layton D, Osborne V, Gilchrist A and Shakir SA (2011). Examining the utilization and tolerability of the non-sedating antihistamine levocetirizine in England using prescription-event monitoring data. Drug Saf. 34: 1177-1189.

Navarro A, Valero A, Rosales MJ and Mullol J (2011). Clinical use of oral antihistamines and intranasal corticosteroids in patients with allergic rhinitis. J. Investig. Allergol. Clin. Immunol. 21: 363-369.

Otorhinolaryngology Society of the Chinese Medical Association (2005). The Editorial Committee of Chinese Journal of Otorhinolaryngology Head and Neck Surgery. The Principles of Diagnosis and Treatment of Allergic Rhinitis and Recommended Plan (2004, Lanzhou, China). Chin. J. Otorhinolaryngol. Head Neck Surgery 40: 166.

Pasquali M, Baiardini I, Rogkakou A, Riccio AM, et al. (2006). Levocetirizine in persistent allergic rhinitis and asthma: effects on symptoms, quality of life and inflammatory parameters. Clin. Exp. Allergy 36: 1161-1167.

Ribeiro de Andrade C, Chatkin JM, Fiterman J, Scaglia N, et al. (2010). Unified disease, unified management: treating allergic rhinitis and asthma with nasally inhaled corticosteroid. Respir. Med. 104: 1577-1580.

von Bernus L, Högger P, Pfaar O and Klimek L (2012). Mechanism of action of nasal glucocorticosteroids in the treatment of allergic rhinitis. Part 2: Practical aspects of application. HNO 60: 700-706.

Vovolis V, Kalogiros L, Mitsias D and Sifnaios E (2013). Severe repeated anaphylactic reactions to sublingual immunotherapy. Allergol. Immunopathol. 41: 279-281.

Wu F, Chen WZ, Bai J, Zou JZ, et al. (2001). Pathological changes in human malignant carcinoma treated with high intensity focused ultrasound. Ultrasound Med. Biol. 27: 1099-1106. 